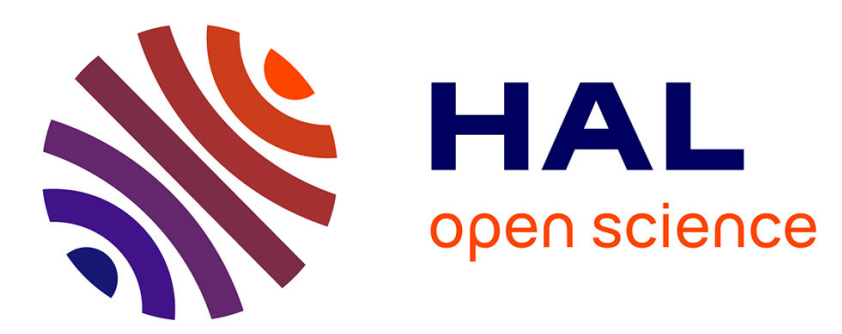

\title{
Alkaline-earth metal monolayers on fivefold i-Al-Pd-Mn surface: Influence of adatom size on quasiperiodic ordering
}

\author{
Marian Krajci, Juergen Hafner
}

\section{- To cite this version:}

Marian Krajci, Juergen Hafner. Alkaline-earth metal monolayers on fivefold i-Al-Pd-Mn surface: Influence of adatom size on quasiperiodic ordering. Philosophical Magazine, 2008, 88 (13-15), pp.21172122. 10.1080/14786430801995135 . hal-00513878

\section{HAL Id: hal-00513878 \\ https://hal.science/hal-00513878}

Submitted on 1 Sep 2010

HAL is a multi-disciplinary open access archive for the deposit and dissemination of scientific research documents, whether they are published or not. The documents may come from teaching and research institutions in France or abroad, or from public or private research centers.
L'archive ouverte pluridisciplinaire HAL, est destinée au dépôt et à la diffusion de documents scientifiques de niveau recherche, publiés ou non, émanant des établissements d'enseignement et de recherche français ou étrangers, des laboratoires publics ou privés. 


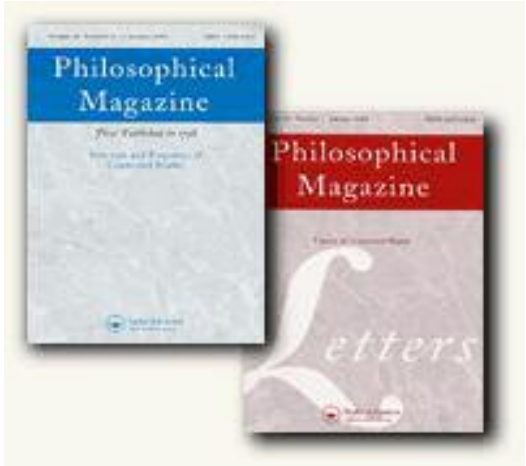

\section{Alkaline-earth metal monolayers on fivefold i-Al-Pd-Mn surface: Influence of adatom size on quasiperiodic ordering}

\begin{tabular}{|r|l|}
\hline Journal: & Philosophical Magazine \& Philosophical Magazine Letters \\
\hline Manuscript ID: & TPHM-07-Oct-0279.R1 \\
\hline $\begin{array}{r}\text { Dournal Selection: } \\
\text { Author: }\end{array}$ & Philosophical Magazine \\
\hline Complete List of Authors: & $\begin{array}{l}\text { Krajci, Marian; Slovak Academy of Sciences, Institute of Physics; } \\
\text { University of Vienna, Center for Computational Materials Science } \\
\text { Hafner, Juergen; University of Vienna, Faculty of Physics, Center for } \\
\text { Computational Materials Science }\end{array}$ \\
\hline Keywords: & ab initio, adsorption, quasicrystals, surfaces \\
\hline Keywords (user supplied): & Al-Pd-Mn, alkaline-earth metals \\
\hline & \\
\hline $\begin{array}{l}\text { Note: The following files were submitted by the author for peer review, but cannot be converted } \\
\text { to PDF. You must view these files (e.g. movies) online. }\end{array}$ \\
\hline monolayers2.tex \\
\hline
\end{tabular}

\section{S) ScholarONE" \\ Manuscript Central}




\title{
Alkaline-earth metal monolayers on fivefold i-Al-Pd-Mn surface: Influence of adatom size on quasiperiodic ordering
}

\author{
M. KRAJČíl† ${ }^{\dagger} \ddagger$ and J. HAFNER $\dagger$ \\ $\dagger$ Faculty of Physics, CCMS, University of Vienna, \\ Sensengasse 8/12, A-1090 Vienna, Austria \\ $¥$ Institute of Physics, Slovak Academy of Sciences, \\ Dúbravská cesta 9, SK-84511 Bratislava, Slovak Republic
}

The structure and stability of a quasiperiodic alkaline-earth metal monolayers formed on a five-fold surface of an icosahedral Al-Pd-Mn quasicrystal have been investigated using ab-initio density-functional methods. We study an influence of the size of adatom on the regularity of the quasiperiodic ordering. It was found that $\mathrm{Ca}$ adatoms at the coverage 0.066 atoms $/ \AA^{2}(\Theta=0.5)$ form on the i-Al-Pd-Mn surface a highly regular quasiperiodic monolayer with an ordering described by a decagonal DHBS tiling of decagons, hexagons, boats and pentagonal stars.

Keywords: quasicrystals; surfaces; ab-initio; Al-Pd-Mn; alkaline-earth metals

\section{Introduction}

In our previous works $[1,2,3,4]$ we have investigated the structure and stability of quasiperiodic monolayers by ab initio density-functional methods. One of the important results was an observation that an unsupported quasiperiodic monolayer is not a structurally stable system. A quasiperiodic ordering in a monolayer can be stabilized only when it is supported by a quasicrystalline substrate. A decisive factor determining the structure of an adsorbed monolayer is the density of atoms in the monolayer, or coverage of the surface. The coverage is usually expressed by the parameter $\Theta$ defined as a ratio of the number of adatoms to the number of substrate atoms in the first substrate layer. Depending on the density of the adsorbed adatoms different surface structures are formed. For instance alkali metal atoms on a simple metal surface such as, e.g., $\mathrm{Al}(111)$ can form several phases with complex structures [5]. One can expect even richer variety of adsorbate phases formed on the quasicrystalline substrate. The subject of our interest is an arrangement of atoms in a monolayer with the coverage close to the saturation. The most important factor limiting the atomic density of the adlayer is the size of the adatoms.

In Ref. [3, 4] we studied a formation of alkali metal monolayers and multilayers on the fivefold i-Al-Pd-Mn surface. We have found that $\mathrm{Na}$ and $\mathrm{K}$ adatoms at the coverage $\Theta=0.50\left(0.066\right.$ atoms $\left./ \AA^{2}\right)$ spontaneously arranged in a structure with a quasiperiodic ordering. In the present contribution we extend the study to alkalineearth metals $\mathrm{Be}, \mathrm{Mg}, \mathrm{Ca}, \mathrm{Sr}$ and $\mathrm{Ba}$. These atoms exhibit similar chemical properties but differ significantly in their size. The size of alkaline-earth atoms ranges from the smallest size of $B e$ atom of $2.30 \AA$ to the largest $B a$ atom with the size of $4.35 \AA$. The sequence of the alkaline-earth atoms is thus a suitable system for studying the effect of the density of adatoms on the regularity of the quasiperiodic ordering in the monolayer. For a characterization how dense the monolayer is we introduce also a parameter $\kappa$ expressing the packing of atoms in the monolayer in comparison to the densest planar

\footnotetext{
${ }^{1}$ Corresponding author: Email: fyzikraj@savba.sk
} 
packing the hexagonal close packing. The parameter $\kappa$ for atoms of a given size is calculated as a ratio of the density of atoms in the monolayer to the density of atoms of the same size in a monolayer with the hexagonal close packing. The sizes of atoms are estimated from the nearest-neighbor distances in crystalline low-temperature phases. For hard spheres in a plane with the closest hexagonal packing $\kappa=1.0$. As atoms do not behave as hard spheres and as a monolayer always has certain corrugation, values of $\kappa>1$ are also possible.
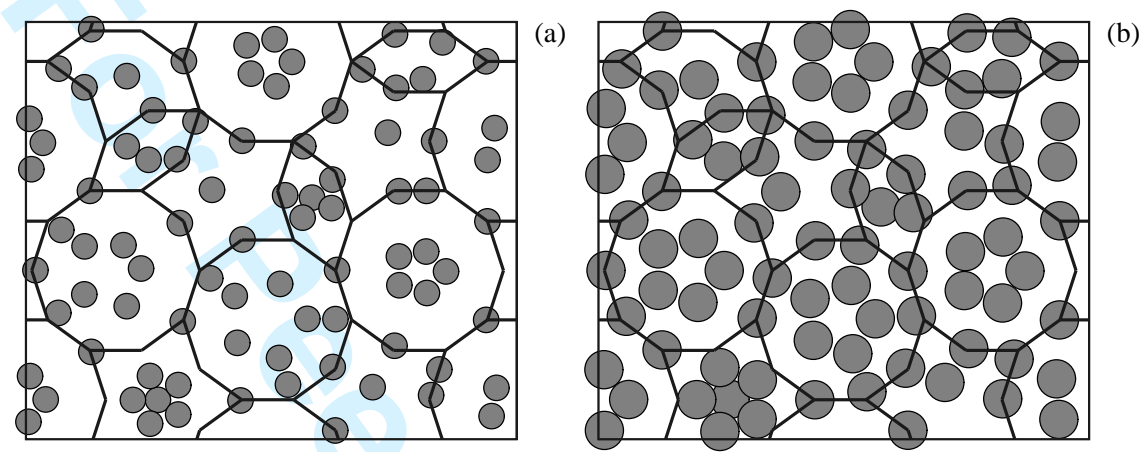

Figure 1: A comparison of the equilibrium arrangements of atoms in $\mathrm{Be}$ (a) and $\mathrm{Mg}$ (b) monolayers on a fivefold i-Al-Pd-Mn surface. The quasiperiodic ordering in the monolayers can be described by the DHBS tiling. Both monolayers are underpacked.

\section{The fivefold surface of i-Al-Pd-Mn}

A model of the atomic structure of the surface is based on a structural model of bulk $\mathrm{i}-\mathrm{Al}-\mathrm{Pd}-\mathrm{Mn}$. A construction of the structural model of the bulk and the fivefold surface derived from periodic approximants to the quasicrystal has been presented in detail in our previous works $[6,7]$. An ideal structure of the fivefold i-Al-Pd-Mn surface consists [8] of two closely spaced atomic planes separated by a vertical distance of $0.48 \AA$. The top plane of the surface is occupied only by $\mathrm{Al}$ atoms and a few percent of $\mathrm{Mn}$ atoms $[9,10]$. Pd atoms from the plane located $0.48 \AA$ below the top layer also contribute to the surface charge density. The corrugated surface is thus composed by the atoms from the two top-most planes. A quasiperiodic ordering in the top atomic plane is most naturally described by a DHBS tiling consisting of decagons (D), squashed hexagons $(H)$, boat tiles $(B)$ and pentagonal stars $(S)$. The edge of the DHBS tiling measures $4.079 \AA$. The vertices of the DHBS tiling are hollows sites surrounded by complete or incomplete $\mathrm{Al}$ pentagons. The total surface atomic density of the model is $\mathrm{n}_{s}=0.132$ atoms $/ \AA^{2}$. This value is in a very good agreement with the experimental value of 0.136 atoms $/ \AA^{2}$ reported by Gierer et al. [9].

All studies of ordering of atoms in monolayers adsorbed on the fivefold surface of $\mathrm{i}-\mathrm{Al}-\mathrm{Pd}-\mathrm{Mn}$ were preformed on the model of the $3 / 2$ approximant [6]. The structural model of the surface used in our adsorption studies has a form of a slab. The slab consists of 5 atomic planes and includes 357 atoms. The size of the slab is 38.63 
$\AA \times 32.86 \AA \times 4.08 \AA$. The computational cell has an orthorhombic shape. In addition to the slab of atoms it includes a $14 \AA$ thick vacuum layer. The binding energies and the interatomic forces have been calculated using the Vienna ab-initio simulation package VASP $[11,12]$.

Very helpful in the search for the structure of the adlayer is a mapping of the energetic landscape of the surface, searching for the most stable positions of adsorbed atoms. As a probe we have chosen a single atom bound to specific sites on the surface. We calculated the binding energy of adsorbed alkaline-earth metal atoms. A systematic repetition of this procedure for selected points on the surface makes it possible to identify the most stable positions for the adsorbed atoms. An equilibrium structure of the adsorbed monolayer has been obtained by relaxing all interatomic forces in the system.

\section{Alkaline-earth monolayers}

Be atom with its size of $2.30 \AA$ is a small atom. A Be monolayer on the fivefold i-Al-Pd-Mn surface at the coverage of $\Theta=0.50$ with the parameter $\kappa=0.30$ is thus clearly underpacked. An ordering of Be atoms on the i-Al-Pd-Mn surface at higher coverages can be very complex. In this work we restrict our study to the case the coverage $\Theta=0.50$ only. The behavior of Be atoms in this underpacked monolayer is remarkable. The lateral cohesive interaction between atoms leads to clustering and chaining the atoms. The equilibrium positions substantially deviate from the ideal positions. Nevertheless, the diffraction pattern shows that the structure still posses a substantial degree of the decagonal symmetry. The diffraction peaks with higher $|\vec{k}|$ have reduced amplitudes. The deviations of the positions of Be atoms from the ideal ones has on the diffraction pattern an analogous effect as the Debye-Waller factor expressing the thermal disorder in ordinary crystals.
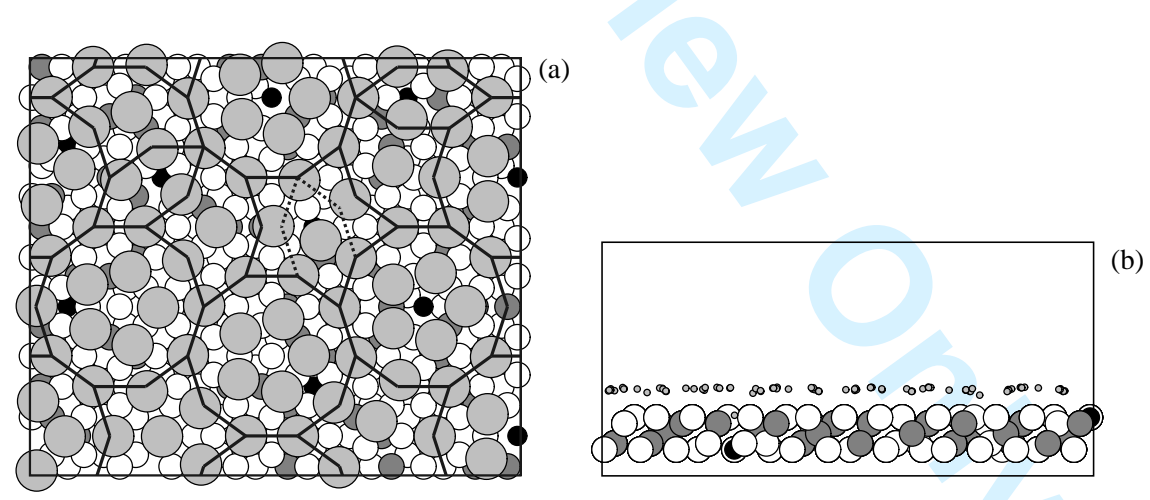

Figure 2: (a) The atomic structure of the Ca monolayer adsorbed on the fivefold i-Al-Pd-Mn surface. The positions of atoms are displayed by circles: $\mathrm{Ca}$ - large shaded circles, Al - small open circles, Pd - small shaded circles, Mn - small solid circles. The surface is covered by the DHBS tiling. Part (b) is a side view on the corresponding structural model demonstrating a corrugation of the Ca monolayer. The centers of $\mathrm{Ca}$ adatoms are represented by small circles. 
A Mg atom with its size of $3.20 \AA$ is substantially bigger than Be. However, the parameter $\kappa$ of 0.59 indicates that the $\mathrm{Mg}$ monolayer is still underpacked. Some of the $\mathrm{Mg}$ adatoms are off their ideal positions. Although the quasiperiodic DHBS distribution of adatoms exhibits a rather high regularity in reality such underpacked structure can be easily distorted by intersticials. The size of $\mathrm{Ca}$ atom of $3.95 \AA$ is similar to that of $\mathrm{Na}$. One can therefore expect a similar highly regular DHBS ordering. Fig. 2(a) shows an equilibrium structure of $\mathrm{Ca}$ monolayer on the i-Al-Pd-Mn substrate. The quasiperiodic ordering at $\Theta=0.50$ and $\kappa=0.89$ is indeed highly regular. The reason why $\mathrm{Ca}$ adatoms spontaneously arrange in a monolayer with the DHBS ordering is possible to understand. The surface plane is Al rich. The top plane consists almost entirely (96\%) of Al atoms. Their geometrical arrangement can be described by the DHBS tiling. It was also found that the top of Al atoms (ALX sites) are the least attractive adsorption sites. While the binding energies of majority of adsorption sites (with an exception of the strongly attractive sites inside surface vacancies) ranges from $-2.7 \mathrm{eV}$ to $-2.2 \mathrm{eV}$ the binding energies of the $\mathrm{ALx}$ sites is $\approx-1.35 \mathrm{eV}$. Adatoms thus prefer to occupy all other sites except the ALx ones. This forces the adatoms to correlate their ordering with the ordering of the distribution of the $\mathrm{Al}$ atoms in the top atomic plane and hence to adopt the DHBS ordering. In Fig. 2 it is seen that $\mathrm{Ca}$ adatoms avoid the positions on top of $\mathrm{Al}$ atoms with lowest binding energies.
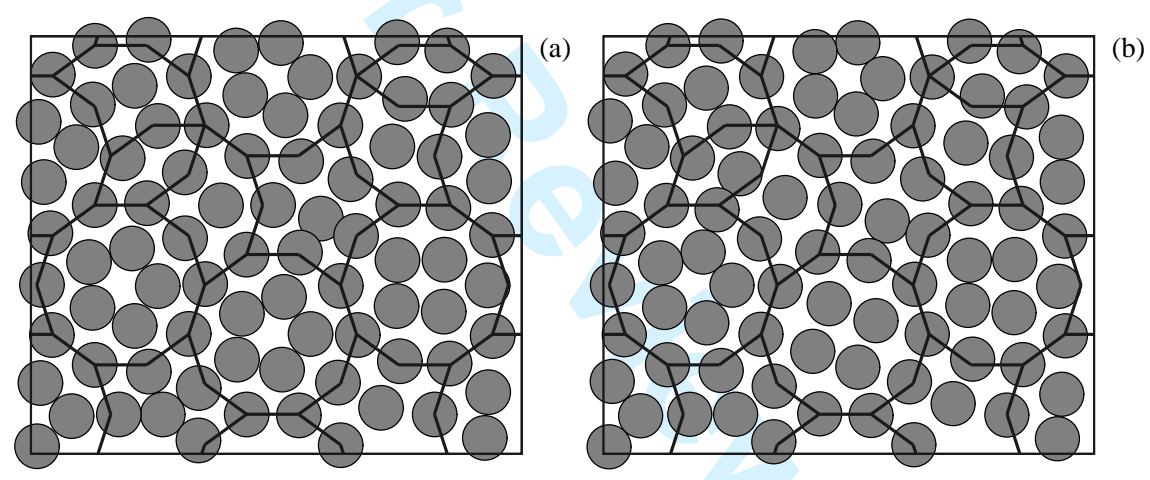

Figure 3: A comparison of the equilibrium arrangements of atoms in $\mathrm{Sr}$ (a) and $\mathrm{Ba}$ (b) monolayers on a fivefold i-Al-Pd-Mn surface. The quasiperiodic ordering in the monolayers can be described by the DHBS tiling. Both Sr and Ba monolayer are overpacked. A larger size of Sr and Ba atoms leads to a breaking of pentagonal symmetry of the atomic packing inside of some of the decagonal tiles. One $\mathrm{Ba}$ adatom drifted off the monolayer plane.

The size of $\mathrm{Sr}$ and $\mathrm{Ba}$ atoms of $4.30 \AA$ and $4.35 \AA$, respectively, leads at the coverage $\Theta=0.50$ to the packing $\kappa=1.06$ and 1.08 , respectively, i.e. to a slight overpacking. The overpacking results to a higher corrugation of the monolayer and to distortion of the pentagonal symmetry of the internal decoration of the $D$ tiles. One adatom in the Ba monolayer drifted off the monolayer plane. There is one interesting detail in the DHBS tiling. When one compares the DHBS tiling in the $\mathrm{Mg}, \mathrm{Ca}, \mathrm{Sr}$, and $\mathrm{Ba}$ monolayers in Figs. 1 to 3 it is seen that while the ordering of $\mathrm{Mg}$ atoms near the center of the figure is described by one $\mathrm{H}$ and two $\mathrm{S}$ tiles the ordering of $\mathrm{Sr}$ and $\mathrm{Ba}$ 
atoms in that area is described by one $S$ and two $B$ tiles. This can be understood as a phason flip (dashed lines in Fig. 2(a)) in the tiling caused by shift of positions of two atoms between two close sites. In the Ca monolayer the corresponding adatoms remain in a "midway" between these two positions.

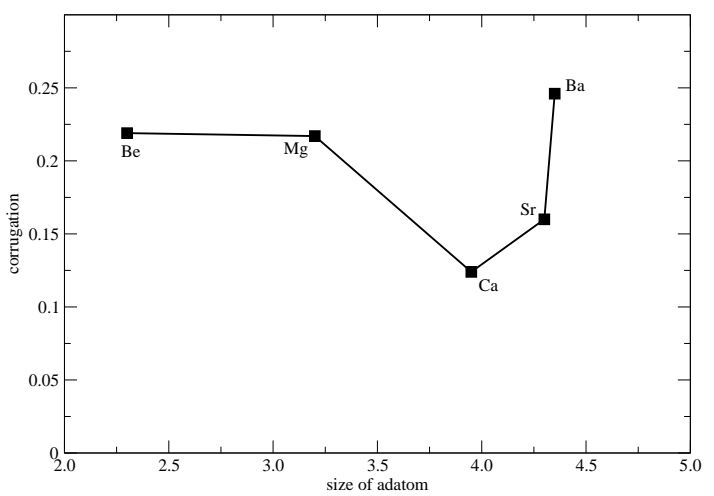

Figure 4:

A corrugation of the quasiperiodic monolayer expressed by mean deviation $\sigma[\AA]$ for various adatoms, see Tab. 1 . The horizontal axis is the size of adatoms in $\AA$.

\section{Discussion}

The size of the adatoms and the coverage density have appeared to be the most important factors determining regularity of the monolayer. An optimal size of the adatoms can lead to a sufficiently high packing density enforcing a regular arrangement of atoms. In an underpacked monolayer one can expect formation of interstitial sites that spoil regularity of the ideal quasiperiodic structure. A good measure of regularity of the monolayer is its corrugation. The corrugation is expressed as a mean deviation $\sigma$ of the vertical positions of adatoms from their average height above the surface plane. The corrugations of monolayers consisting of small adatoms like $\mathrm{Be}$ or $\mathrm{Mg}$ copy the corrugation of the substrate. The amplitude of this corrugation can be estimated as $\sigma \approx 0.22 \AA$. On the other hand a large size of the adatoms leads to an overpacking of the monolayer and its corrugation also increases, e.g. $\sigma \approx 0.25 \AA$ in the Ba monolayer. The corrugation is the lowest at certain "optimal" size of adatom, see Fig. 4. The Ca monolayer with the size of adatoms of $3.95 \AA$ exhibits the lowest corrugation $\sigma=0.12$ $\AA$. The size of $\mathrm{Ca}$ adatoms is most compatible with the geometrical distribution of adsorption sites on the fivefold i-Al-Pd-Mn surface and hence the quasiperiodic ordering in the $\mathrm{Ca}$ monolayer is most regular. 
Table 1: Structural data of the studied monolayers: Size of adatom $d$, an average height $h$ of the atoms in the adsorbed monolayer above the surface plane, a corrugation of the monolayer $\sigma$, a relative corrugation $\sigma / h$, and the parameter $\kappa$ expressing the packing of adatoms in the monolayer in comparison to the densest hexagonal close packing.

\begin{tabular}{lcccccc} 
Monolayer & $d[\AA]$ & $h[\AA]$ & $\sigma[\AA]$ & $\sigma / h$ & $\kappa$ & see Fig. \\
\hline $\mathrm{Be}$ & 2.30 & 1.14 & 0.219 & 0.192 & 0.30 & $1(\mathrm{a})$ \\
$\mathrm{Mg}$ & 3.20 & 1.79 & 0.217 & 0.121 & 0.59 & $1(\mathrm{~b})$ \\
$\mathrm{Ca}$ & 3.95 & 2.20 & 0.124 & 0.056 & 0.89 & 2 \\
$\mathrm{Sr}$ & 4.30 & 2.44 & 0.160 & 0.066 & 1.06 & $3(\mathrm{a})$ \\
$\mathrm{Ba}$ & 4.35 & 2.55 & 0.246 & 0.096 & 1.08 & $3(\mathrm{~b})$ \\
\hline
\end{tabular}

\section{Acknowledgment}

This work has been supported by the Austrian Ministery for Education, Science and Art through the Center for Computational Materials Science (CCMS). M. K. thanks also for support from from the grants No. VEGA-2/0157/08, APVV-0413-06 and CEX-Nanosmart.

\section{References}

[1] M. Krajčí and J. Hafner, Phys. Rev. B. 71, 184207 (2005).

[2] M. Krajčí, J. Hafner, and M. Jahnátek, Phys. Rev. B. 73, 184202 (2006).

[3] M. Krajčí and J. Hafner, Phys. Rev. B. 75, 224205 (2007).

[4] M. Krajčí and J. Hafner, Phil Mag. 87, 2981 (2007).

[5] R. D. Diehl, and R. McGrath, Surf. Sci. Reports 23, 43 (1996).

[6] M. Krajčí and J. Hafner, Phys. Rev. B. 71, 054202 (2005).

[7] M. Krajčí, J. Hafner, J. Ledieu, and R. McGrath, Phys. Rev. B. 73, 024202 (2006).

[8] Z. Papadopolos, P. Pleasants, G. Kasner, V. Fournée, C.J. Jenks, J. Ledieu, and R. McGrath, Phys. Rev. B 69, 224201 (2004).

[9] M. Gierer, M.A. Van Hove, A.I. Goldman, Z. Shen, S.-L. Chang, P.J. Pinhero, C.J. Jenks, J.W. Anderegg, C.-M. Zhang, and P.A. Thiel, Phys. Rev. B 57, 7628 (1998).

[10] J.C. Zheng, C.H.A. Huan, A.T.S. Wee, M.A. Van Hove, C.S. Fadley, F.J. Shi, E. Rotenberg, S.R. Barman, J.J. Paggel, K. Horn, P. Ebert, and K. Urban, Phys. Rev. B 69, 134107 (2004).

[11] G. Kresse and J. Furthmüller, Comp. Mat. Sci. 6, 15 (1996); Phys. Rev. B 54, 11169 (1996).

[12] G. Kresse and D. Joubert, Phys. Rev. B 59, 1758 (1999). 

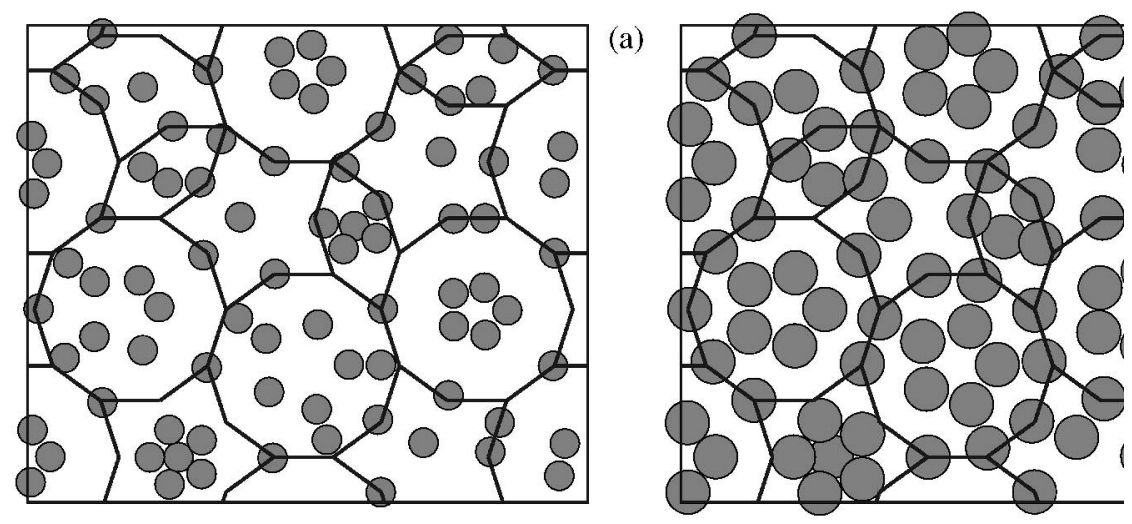

Figure 1

$215 \times 279 m m(600 \times 600$ DPI $)$ 

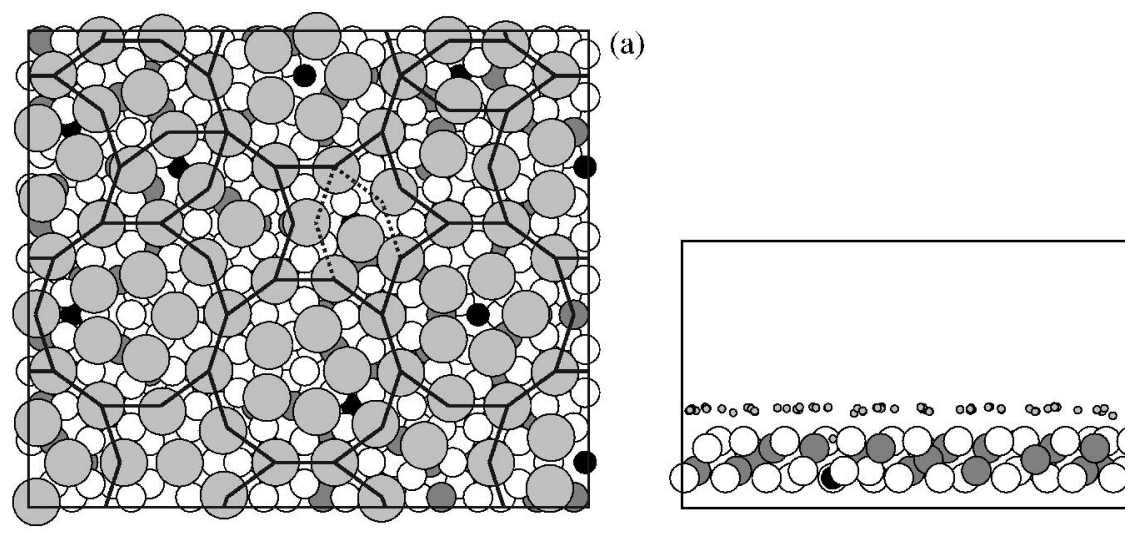

Figure 2

$215 \times 279 \mathrm{~mm}(600 \times 600$ DPI $)$ 

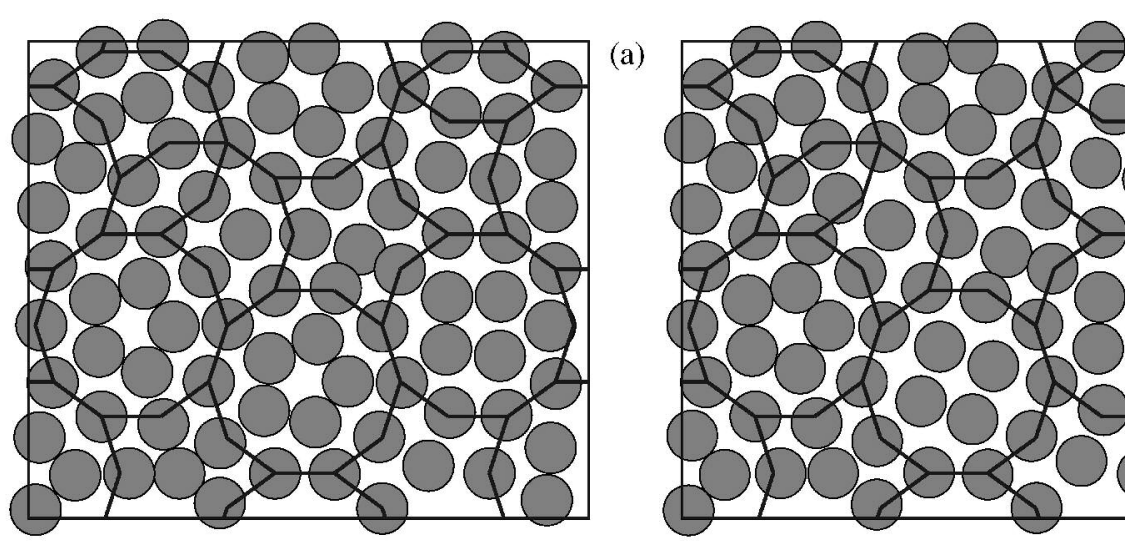

Figure 3

$215 \times 279 \mathrm{~mm}(600 \times 600 \mathrm{DPI})$ 


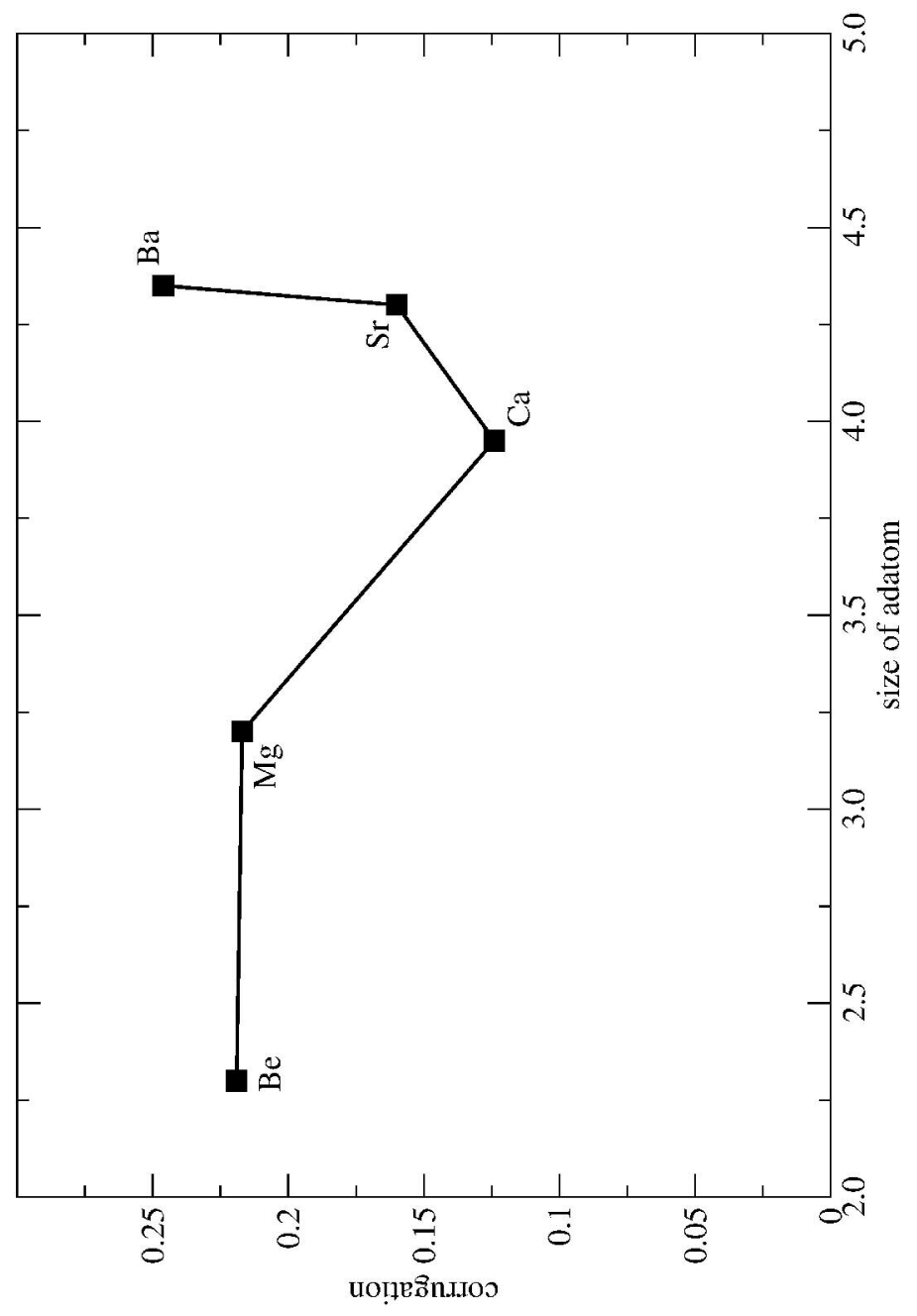

Figure 4

$215 \times 279 \mathrm{~mm}(600 \times 600 \mathrm{DPI})$ 